\title{
Complementarity Active-Set Algorithm for Mathematical Programming Problems with Equilibrium Constraints
}

\author{
J.J. Júdice · H.D. Sherali • I.M. Ribeiro •
}

A.M. Faustino

Published online: 21 July 2007

(C) Springer Science+Business Media, LLC 2007

\begin{abstract}
In this paper, an algorithm for solving a mathematical programming problem with complementarity (or equilibrium) constraints (MPEC) is introduced, which uses the active-set methodology while maintaining the complementarity restrictions throughout the procedure. Finite convergence of the algorithm to a strongly stationary point of the MPEC is established under reasonable hypotheses. The algorithm can be easily implemented by adopting any active-set code for nonlinear programming. Computational experience is included to highlight the efficacy of the proposed method in practice.
\end{abstract}

Keywords Local optimization · Complementarity · Mathematical programming problem with equilibrium constraints $\cdot$ Active-set algorithm

Communicated by P.M. Pardalos.

Support by Instituto de Telecomunicações, FCT Grant POCTI/35059/MAT/2000, by National Science Foundation Grant DMI-0094462 and by FCT_-Fundação para a Ciência e Tecnologia, Portugal.

J.J. Júdice

Departamento de Matemática, Universidade de Coimbra, Coimbra, Portugal

e-mail: judice@mat.uc.pt

J.J. Júdice

Instituto de Telecomunicações, Lisboa, Portugal

H.D. Sherali

Grado Department of Industrial \& Systems Engineering, Virginia Polytechnic Institute,

Blacksburg, VA, USA

H.D. Sherali

Virginia State University, Blacksburg, VA, USA

I.M. Ribeiro $(\varangle) \cdot$ A.M. Faustino

Secção de Matemática do Departamento de Engenharia Civil, Faculdade de Engenharia,

Universidade do Porto, Porto, Portugal

e-mail: iribeiro@fe.up.pt 


\section{Introduction}

A mathematical programming problem with equilibrium constraints (MPEC) is an optimization problem in which the constraints include complementarity restrictions or parametric variational inequalities. Complementarity problems and variational inequalities are related typically with phenomena of balance that appear in several important practical applications in engineering and economy. MPECs can be viewed as extensions of bilevel programs and are also known as mathematical programs with optimization constraints. A detailed study on this type of problems is presented in $[1,2]$ and number of algorithms are described in [1-9].

The objective of the present paper is to develop a local solution algorithm for MPECs when the constraint functions are linear. Thus, we focus on the following problem

$$
\begin{aligned}
\text { (MPEC) } \min & f(y, z, w), \\
\text { s.t. } & E w=q+M z+N y, \quad y \in K_{y}, \\
& z \geq 0, \quad w \geq 0, \quad z^{T} w=0,
\end{aligned}
$$

where $q \in \mathbb{R}^{l}, z, w \in \mathbb{R}^{n}, y \in \mathbb{R}^{m}, E \in \mathbb{R}^{l \times n}, M \in \mathbb{R}^{l \times n}, N \in \mathbb{R}^{l \times m}, f: \mathbb{R}^{m \times n} \rightarrow \mathbb{R}$ is twice continuously differentiable in an open set that contains the feasible set associated with (1) and $K_{y} \subseteq \mathbb{R}^{m}$ is a convex polyhedron in $y$. In many applications of MPEC, the matrices $E$ and $M$ are square, with $E$ an identity matrix and $M$ a positivesemidefinite (PSD) matrix, or the problem can be transformed into an MPEC having these properties.

Contrary to the approaches mentioned above, we exploit directly the generalized linear complementarity problem (GLCP) given the constraints of the MPEC (1). As in [10], the approach to be discussed in this paper uses the active-set methodology and maintains complementarity during the entire procedure. Finite termination for the procedure to a strongly stationary point [8] of the MPEC is established under a nondegeneracy assumptions. A number of improvements of the procedure are introduced in order to deal with degenerate cases. As the active-set algorithm is based on the use of complementary solutions, the solution of the GLCP associated with the MPEC is a key point for the algorithm. This topic is also addressed in this paper. Computational experience is included to highlight the efficiency of the algorithm in dealing with MPECs related to bilevel problems [11], NP-hard LCPs through their equivalence to bilinear programs [12], and from other known sources [6].

In Sect. 2, a complementarity active-set algorithm (CASET) for finding a strongly stationary point for MPEC is discussed in detail. The convergence of CASET is established in Sect. 3. The implementation of CASET as well as degenerate cases are treated in Sect. 4. Finally, we conclude the paper with some computational experience and remarks on implementing the proposed algorithm.

\section{Complementarity Active-Set Algorithm}

In this section, we discuss in detail a complementarity active-set algorithm (CASET) for finding a strongly stationary point for MPEC, that is, a solution satisfying the necessary first-order KKT conditions of the nonlinear program (NLP), which is ob- 
tained from MPEC (1) by considering the complementarity conditions $z_{i} w_{i}=0$, $i=1, \ldots, n$, as constraints. Thus, this NLP has the following form:

$$
\begin{aligned}
(\mathrm{NLP}) \min & f(y, z, w) \\
\text { s.t. } \quad & E w=q+M z+N y, \quad A y=b, \\
& z \geq 0, \quad w \geq 0, \quad y \geq 0, \\
& z_{i} w_{i}=0, \quad i=1, \ldots, n .
\end{aligned}
$$

The proposed algorithm is similar to a procedure described by Scholtes [10] and consists essentially of using an active-set technique on the set of solutions of the GLCP given by the constraints of the MPEC. Thus, at each iteration $k$, the corresponding solution $(w, z, y)$ satisfies the constraints of (1) and the set of the active constraints at this solution is given by

$$
\begin{aligned}
& E w-M z-N y=q, \\
& A y=b, \\
& w_{i}=0, \quad i \in L_{w} \subseteq\{1, \ldots, n\}, \\
& z_{i}=0, \quad i \in L_{z} \subseteq\{1, \ldots, n\}, \\
& y_{i}=0, \quad i \in L_{y} \subseteq\{1, \ldots, m\},
\end{aligned}
$$

where $L_{z}, L_{y}, L_{w}$ are the sets of the currently active constraints corresponding to the nonnegative constraints on the variables $z, y, w$, respectively and $L_{z} \cup L_{w}=$ $\{1, \ldots, n\}$.

The active constraints (2) constitute a linear system of the form $D_{k} x=g^{k}$ where $x=\left(w^{T}, z^{T}, y^{T}\right)^{T}$ and $D_{k} \in \mathbb{R}^{t \times(2 n+m)}$, with $t=l+p+\left|L_{w}\right|+\left|L_{z}\right|+\left|L_{y}\right|$, and where $|H|$ is the cardinal of the set $\mathrm{H}$. Recall that $p$ and $l$ are the number of rows of the matrices $A$ and $[E,-M,-N]$, respectively. The first-order optimality conditions for the problem

$$
\min \left\{f(x): D_{k} x=g^{k}\right\}
$$

can be written in the form

$$
\nabla f(x)=D_{k}^{T} \mu, \quad D_{k} x=g^{k} .
$$

In order to facilitate a unique set of Lagrange multipliers $\mu$, we assume that the following condition holds throughout the proposed procedure:

Nondegeneracy Assumption $t \leq 2 n+m$ and $\operatorname{rank}\left(D_{k}\right)=t$.

As we show later, this hypothesis is not restrictive under the usual full row rank assumption of the matrices $A$ and $[E,-M,-N]$. Consequently, the active-set is always linearly independent. Furthermore, let us partition the Lagrange multipliers vector $\mu$ into three subvectors denoted by $\beta \rightarrow$ subvector associated with the first set of equality constraints in (2), $\vartheta \rightarrow$ subvector associated with the second set of equality constraints in (2), $\lambda_{i}^{x} \rightarrow$ subvector associated with $x_{i}=0$ in the last three sets of equality constraints in (2). 
The main steps of the complementary active-set algorithm are described below.

\section{Complementarity Active-Set Algorithm-CASET}

Step 0 . Set $k=1$ and find a solution $x^{k}$ of the GLCP associated with MPEC. Let $D_{k} x=g^{k}$ be the set of active constraints at $x^{k}$ and let $L_{y}, L_{z}, L_{w}$ be the index sets associated with the nonnegative active constraints $y_{i}=0, z_{i}=0$, $w_{i}=0$, respectively.

Step 1. Optimality Conditions

If $x^{k}$ is not a stationary (KKT) point (see [13]) for the equality problem

$$
\begin{array}{ll}
\text { (EP) } \min & f(x), \\
\text { s.t. } & D_{k} x=g^{k},
\end{array}
$$

then go to Step 2. Otherwise, there exists a unique $\mu$ such that $D_{k}^{T} \mu=$ $\nabla f\left(x^{k}\right)$ and two cases can occur:

(1.1) If $\lambda_{i}^{y} \geq 0 \forall i \in L_{y}, \lambda_{i}^{z} \geq 0 \forall i \in L_{z} \cap L_{w}$ and $\lambda_{i}^{w} \geq 0 \forall i \in L_{z} \cap L_{w}$, stop; $x^{k}$ is a strongly stationary point for MPEC (see Theorem 3.1 below).

(1.2) If there exists at least one $i$ such that $\lambda_{i}^{y}<0$ for $i \in L_{y}$ or $\lambda_{i}^{z}<0$ for $i \in$ $L_{z} \cap L_{w}$ or $\lambda_{i}^{w}<0$ for $i \in L_{z} \cap L_{w}$, remove an active constraint $y_{i}=$ 0 , or $z_{i}=0$, or $w_{i}=0$, associated with the most negative Lagrange multiplier. Let $D_{k_{i}} x=g_{i}^{k}$ be the row removed from $D_{k} x=g^{k}$ and rearrange the rows of $D_{k} x=g^{k}$ in the following way:

$$
D_{k}=\left[\begin{array}{c}
\bar{D}_{k} \\
D_{k_{i}}
\end{array}\right], \quad g^{k}=\left[\begin{array}{c}
\bar{g}^{k} \\
g_{i}^{k}
\end{array}\right] .
$$

Find a direction $d$ such that $\nabla f\left(x^{k}\right)^{T} d<0, \bar{D}_{k} d=0$, and $D_{k_{i}} d>0$ (Theorem 3.2 establishes below that such $d$ exists). Replace $D_{k}$ by $\bar{D}_{k}$ and go to Step 3.

\section{Step 2. Determination of Search Direction}

Find a descent direction for $f$ in the set of active constraints, i.e., find $d$ such that

$$
\nabla f\left(x^{k}\right)^{T} d<0, \quad D_{k} d=0 .
$$

\section{Step 3. Determination of Stepsize}

(3.1) Find the largest value $\alpha_{\max }$ of $\alpha$ such that $x^{k}+\alpha d \geq 0$, i.e.,

$$
\alpha_{\max }=\min \left\{\frac{x_{i}^{k}}{-d_{i}}: d_{i}<0, i \notin\left(L_{y} \cup L_{z} \cup L_{w}\right)\right\} .
$$

(3.2) Compute $0<\alpha_{k} \leq \alpha_{\max }$ such that $x^{k}+\alpha_{k} d$ provides a sufficient decrease for $f$ using any line search technique [13]. If $\alpha_{k}=+\infty$, stop; MPEC is unbounded. 
Step 4. Update of Iterate

Compute $x^{k+1}=x^{k}+\alpha_{k} d$. If $\alpha_{k}=\alpha_{\max }$, add to the active set the constraint $x_{i} \geq 0$ for which $\alpha_{\max }$ was attained such that the nondegeneracy assumption remains true. Return to Step 1.

\section{Convergence of the Complementarity Active-Set Algorithm}

In this section, the convergence of the complementarity active-set algorithm to a strongly stationary point for MPEC is proved. To begin, we establish a sufficient condition for a stationary point to problem (4) in Step 1 to be a strongly stationary point for MPEC.

Theorem 3.1 At any iteration $k$, let $x^{k}$ be a stationary (KKT) point with Lagrange multipliers $\mu$ for the problem

$$
\min \left\{f(x): \quad D_{k} x=g^{k}\right\}
$$

where $D_{k} x=g^{k}$ represents all the active constraints of MPEC at $x^{k}$ and the $x^{k}$-components for the inactive constraints are positive. If $\lambda_{i}^{y} \geq 0, \forall i \in L_{y}$, and $\left(\lambda_{i}^{z}, \lambda_{i}^{w}\right) \geq 0, \forall i \in L_{z} \cap L_{w}$, then $x^{k}$ is a strongly stationary point for MPEC.

Proof By definition, $x^{k}$ is feasible to the linear constraints of MPEC. Moreover $x^{k}$ satisfies the complementarity constraints, since $L_{z} \cup L_{w}=\{1, \ldots, n\}$ in each iteration. By the KKT conditions for (5), we have

$$
D_{k}^{T} \mu=\nabla f\left(x^{k}\right)
$$

Hence, to prove that $x^{k}$ is a strongly stationary point for MPEC, we need to show that there exist $\bar{\mu}$ and $\bar{\psi}$ such that

$$
\begin{aligned}
& D_{k}^{T} \bar{\mu}+\sum_{i=1}^{n}\left[\xi_{i}\right] \bar{\psi}_{i}=\nabla f\left(x^{k}\right), \\
& \bar{\lambda}_{i}^{y} \geq 0, \quad \forall i \in L_{y}, \quad \bar{\lambda}_{i}^{z} \geq 0, \quad \forall i \in L_{z}, \quad \bar{\lambda}_{i}^{w} \geq 0, \quad \forall i \in L_{w},
\end{aligned}
$$

where the variables $\bar{\lambda}_{i}^{y}, \bar{\lambda}_{i}^{z}, \bar{\lambda}_{i}^{w}$ are the components of $\mu$ as defined above, and where

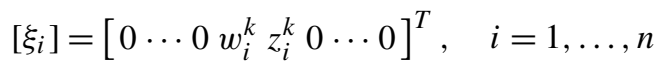

$$
\begin{aligned}
& \uparrow \uparrow \\
& \text { position for } z_{i} \text { position for } w_{i}
\end{aligned}
$$

and $\bar{\psi}_{i}$ is the Lagrange multiplier associated with the constraint $z_{i} w_{i}=0, i=$ $1, \ldots, n$.

Now, let us define $\bar{\mu}$ to have the same components as $\mu$ for the first two sets of equalities in (2), the remaining components of $\bar{\mu}$ being given as follows: 


$$
\begin{aligned}
& \bar{\lambda}_{i}^{y}=\lambda_{i}^{y}, \quad \forall i \in L_{y}, \\
& \bar{\lambda}_{i}^{z}= \begin{cases}\lambda_{i}^{z}, & \text { if } \lambda_{i}^{z} \geq 0, \\
0, & \text { otherwise, } \forall i \in L_{z},\end{cases} \\
& \bar{\lambda}_{i}^{w}= \begin{cases}\lambda_{i}^{w}, & \text { if } \lambda_{i}^{w} \geq 0, \\
0, & \text { otherwise, } \forall i \in L_{w},\end{cases} \\
& \bar{\psi}_{i}= \begin{cases}\frac{\lambda_{i}^{z}}{w_{i}^{k}}, & \text { if } \lambda_{i}^{z}<0, \\
\frac{\lambda_{i}^{w}}{z_{i}^{k}}, & \text { if } \lambda_{i}^{w}<0, \\
0, & \text { otherwise, } \forall i=1, \ldots, n .\end{cases}
\end{aligned}
$$

Then, (7b) is satisfied. Furthermore, note that $\bar{\psi}$ is well defined, since $\left\{i \in L_{z}\right.$ : $\left.\lambda_{i}^{z}<0\right\} \cap\left\{i \in L_{w}: \lambda_{i}^{w}<0\right\}=\emptyset$. In fact:

(i) $\lambda_{i}^{z} \geq 0$ and $\lambda_{i}^{w} \geq 0$ for all $i \in L_{z} \cap L_{w}$.

(ii) $\lambda_{i}^{z}<0 \Rightarrow i \in L_{z} \backslash\left(L_{z} \cap L_{w}\right) \Rightarrow i \notin L_{w} \Rightarrow w_{i}^{k}>0 \Rightarrow \lambda_{i}^{w}=0$ by the hypothesis. (iii) $\lambda_{i}^{w}<0 \Rightarrow \lambda_{i}^{w}=0$, as in (ii).

Now if $\lambda_{i}^{z}<0$, let $e_{i}^{z}$ denote the unit vector in $\mathbb{R}^{n}$ that represents the column of $D_{k}^{T}$ corresponding to $\lambda_{i}^{z}$ in (6). Then, it follows from (8) and (9d) that

$$
\left[e_{i}^{z}\right] \lambda_{i}^{z}=\left[\xi_{i}\right] \bar{\psi}_{i}, \quad \forall i \in L_{z} \text { such that } \lambda_{i}^{z}<0 .
$$

Similarly, considering $i \in L_{w}$ for which $\lambda_{i}^{w}<0$, we get

$$
\left[e_{i}^{w}\right] \lambda_{i}^{w}=\left[\xi_{i}\right] \bar{\psi}_{i}, \quad \forall i \in L_{w} \text { such that } \lambda_{i}^{w}<0 .
$$

Hence, from (6) and from (9a) to (10), we have that $\bar{\mu}$ and $\bar{\psi}$ given by (9) satisfy (7), and this completes the proof.

Note that this theorem also holds if the nondegeneracy condition $x_{i}^{k}>0$ for all inactive $i$ is replaced by $w_{i}^{k}+z_{i}^{k}>0$ for all $i=1, \ldots, n$. However, this latter condition does not assure that $\alpha_{\max }$ in Step 3 is always positive.

Next, we prove that whenever the sufficient conditions identified in Theorem 3.1 are not satisfied, the objective function value of MPEC can be improved via Case 1.2 of Step 1 .

Theorem 3.2 At any iteration $k$, consider the situation described by Theorem 3.1, but suppose that some Lagrange multiplier $\mu_{i}$, say, is negative, where $\mu_{i}$ might be $\lambda_{i}^{y}$ for $i \in L_{y}$, or $\lambda_{i}^{z}$ for $i \in L_{z} \cap L_{w}$, or $\lambda_{i}^{w}$ for $i \in L_{z} \cap L_{w}$. Denote $D_{k_{i}} x=g_{i}^{k}$ as the corresponding row of $D_{k} x=g^{k}$, and rearrange the rows of $D_{k} x=g^{k}$ according to

$$
D_{k}=\left[\begin{array}{c}
\bar{D}_{k} \\
D_{k_{i}}
\end{array}\right], \quad g^{k}=\left[\begin{array}{c}
\bar{g}^{k} \\
g_{i}^{k}
\end{array}\right], \quad \mu_{k}=\left[\begin{array}{c}
\mu^{\prime} \\
\mu_{i}
\end{array}\right] .
$$

Then, the problem

$$
\min \left\{f(x): \bar{D}_{k} x=\bar{g}^{k}\right\}
$$


has a feasible descent direction d satisfying

$$
\nabla f\left(x^{k}\right)^{T} d<0, \quad \bar{D}_{k} d=0, \quad D_{k_{i}} d>0 .
$$

Proof Note that the KKT conditions for (5) assert that

$$
\bar{D}_{k}^{T} \mu^{\prime}+D_{k_{i}}^{T} \mu_{i}=\nabla f\left(x^{k}\right) .
$$

Let $d$ be any solution to the system

$$
\left[\begin{array}{c}
\bar{D}_{k} \\
D_{k_{i}}
\end{array}\right] d=e^{n},
$$

where $e^{n}$ is the $n$th column of the identity matrix of order $n$.

Note that by the nondegeneracy assumption (full row-rank of $D_{k}$ ), such a $d$ exists. Moreover, from (13) and (14), we get

$$
\nabla f\left(x^{k}\right)^{T} d=\mu^{\prime T} \bar{D}_{k} d+\mu_{i} D_{k_{i}} d=\mu_{i}<0 .
$$

Noting that (14) asserts $\bar{D}_{k} d=0$ and $D_{k_{i}} d>0$, we have that (12) holds and this completes the proof.

The following theorem establishes the convergence of the CASET algorithm in a finite number of iterations to a KKT point for MPEC, if it exists.

Theorem 3.3 Consider the algorithm CASET, and assume that the KKT solution to problem (5) in Theorem 3.1 is finitely determined (if it exists) at each iteration. Furthermore, assume that the set of KKT solutions to any such problem (5) takes on only finitely many objective function values. Then, CASET finitely terminates with a strongly stationary point for MPEC or an indication of either infeasibility or unboundedness of MPEC.

Proof If GLCP is infeasible, then so is MPEC. Otherwise, we know that MPEC is feasible. If unboundedness is detected when solving problem (5) at any iteration, then MPEC is unbounded. This follows from the fact that $L_{z} \cup L_{w}=\{1, \ldots, n\}$ is maintained and complementarity is enforced in the procedure. Otherwise, the algorithm returns to Step 1 in a finite number of iterations. At this stage, it either detects a strongly stationary point for MPEC as in Theorem 3.1, or determines a feasible descent direction $d$ satisfying (12) of Theorem 3.2. In the former case, termination occurs. In the latter case, by the condition (12) and since $\alpha_{\max }>0$ by the hypotheses of Theorem 3.1, the next iterate $x^{k+1}$ satisfies $f\left(x^{k+1}\right)<f\left(x^{k}\right)$. This strict descent property, along with the assumption on the finite number of possible objective values taken on by the set of KKT solutions for any combination of active constraints, ensures that any given set of active constraints appears only finitely often at Step 1. As the number of possible active sets is finite, the proof is complete.

A particular case of this theorem is presented below. 
Theorem 3.4 If either $f$ is convex or at each iteration the KKT solution $x^{k} d e-$ termined for problem (5) has the least objective function value, then the algorithm CASET is finitely convergent.

Proof If $f$ is convex, then the KKT solutions to problem (5) have a unique objective value and the proof follows from Theorem 3.3. On the other hand, if the KKT solution to problem (5) that is found at each iteration is a global minimum, then the proof again follows from the argument given in the proof of Theorem 3.3.

Therefore we have shown that the active-set algorithm is able to find a strongly stationary point of the MPEC if the hypotheses of Theorem 3.1 and the nondegeneracy assumption hold. In practice, in order to fulfil this assumption, some of the nonnegativity constraints $i$, such that $x_{i}^{k+1}=0$, may have to keep their inactive status. Therefore cycling may occur. However, well known anticycling techniques are implemented in active-set codes such as MiNOS to prevent cycling in practice. Note that if $f$ is nonconvex, we are not always assured of finding a global minimum for problem (5).

\section{Implementation and Degenerate Cases}

It follows from the description of the complementarity active-set algorithm that an initial feasible solution of the MPEC is required for the algorithm to work. Such a feasible point is a solution of the following generalized complementarity problem (GLCP):

$$
\begin{aligned}
& E w=q+M z+N y, \quad A y=b, \\
& z \geq 0, \quad w \geq 0, \quad y \geq 0, \\
& z_{i} w_{i}=0, \quad i=1, \ldots, n .
\end{aligned}
$$

As discussed in [12], if GLCP is feasible, $E$ is the identity matrix and $M$ is positive semidefinite (PSD), then such a solution can be found as a stationary point of the following nonconvex quadratic program:

$$
\begin{array}{ll}
\min & z^{T} w, \\
\text { s.t. } & E w=q+M z+N y, \quad A y=b, \\
& z \geq 0, \quad w \geq 0, \quad y \geq 0 .
\end{array}
$$

Therefore, the GLCP can be solved by an active-set algorithm. This is also valid if the matrices of the GLCP satisfy these properties after some elementary operations. In the general case, the GLCP can be solved by an enumerative method as described in [12]. It is also possible to implement this enumerative method by using an active-set methodology [14]. Computational experience reported in [14] shows that the active-set version of the enumerative method is in general more efficient that the one described in [12] for finding a solution to the GLCP. Hence we come to the conclusion that a solution of the GLCP can always be found by using an active-set 
code, such as Minos [15]. In order to understand the material of this section, it is important to recall how a solution is represented in the MinOs implementation. Let us write the linear constraints of the GLCP (16) in the form

$$
G x=f, \quad x \geq 0,
$$

where $G$ has full row rank. Any feasible solution for this system (17) has three types of variables, the so-called basic, nonbasic, and superbasic variables [15, 16]. These variables are associated with the following partition of the matrix $G$ :

$$
G=[B, S, N],
$$

where $B$ is a square nonsingular matrix associated with the basic variables, and $S$ and $N$ contain the columns of the superbasic and nonbasic variables, respectively. Furthermore, such a solution is basic if and only if there are no superbasic variables, that is, $S$ does not exist in the partition (18).

Now suppose that a solution $\bar{x}=(\bar{w}, \bar{z}, \bar{y})$ of the GLCP has been found. If $\bar{x}$ satisfies the hypothesis of Theorem 3.1, all the active constraints $\bar{x}_{i}=0$ are associated with the nonbasic variables and the nondegeneracy assumption holds at $\bar{x}$. However, some difficulties may arise when the solution found by the algorithm is degenerate, that is, if there are some basic or superbasic variables $\bar{x}_{i}$ with a zero value. To illustrate this case, consider the following MPEC:

$$
\begin{array}{ll}
\min & f\left(w_{1}, w_{2}, z_{1}, z_{2}, y\right)=-y, \\
\text { s.t. } & {\left[\begin{array}{l}
w_{1} \\
w_{2}
\end{array}\right]=\left[\begin{array}{ll}
0 & 1 \\
1 & 0
\end{array}\right]\left[\begin{array}{l}
z_{1} \\
z_{2}
\end{array}\right]+\left[\begin{array}{l}
1 \\
0
\end{array}\right] y+\left[\begin{array}{c}
0 \\
-1
\end{array}\right],} \\
& z \geq 0, \quad w \geq 0, \quad 0 \leq y \leq 2, \quad z^{T} w=0 .
\end{array}
$$

The enumerative algorithm determines the following solution of the GLCP:

$$
\bar{w}=(0,0)^{T}, \quad \bar{z}=(1,0)^{T}, \quad \bar{y}=0 .
$$

An initial solution $x^{1}$ for the CASET algorithm and the corresponding active constraints $D_{1} x^{1}=g^{1}$ are as below

$$
x^{1}=(0,0,1,0,0), \quad D_{1}=\left[\begin{array}{rrrrr}
1 & 0 & 0 & -1 & -1 \\
0 & 1 & -1 & 0 & 0 \\
1 & 0 & 0 & 0 & 0 \\
0 & 1 & 0 & 0 & 0 \\
0 & 0 & 0 & 1 & 0 \\
0 & 0 & 0 & 0 & 1
\end{array}\right], \quad g^{1}=\left[\begin{array}{r}
0 \\
-1 \\
0 \\
0 \\
0 \\
0
\end{array}\right] .
$$

Therefore, $D^{1} \in \mathbb{R}^{6 \times 5}$ and the nondegeneracy assumption is violated. Note that the nondegeneracy assumption holds if we remove a nonnegativity active constraint from the set of active constraints. However, the choice of the active constraint set is not arbitrary. For the algorithm to be able to make some progress, it is recommended that for each pair of complementary constraints $z_{i}=0$ and $w_{i}=0$ exactly one of them is considered as active. In the previous example this is achieved by considering $y=0$ as inactive. Then $y=0$ and $z_{1}=1$ are the basic variables and all the remaining variables are nonbasic. So the solution is basic, as there are no superbasic variables. Furthermore, it satisfies the nondegeneracy assumption and can be an initial point for 
the CASET algorithm. As the nonnegative active constraints correspond to nonbasic variables, we suggest the following process to be applied, if necessary, before the Algorithm CASET comes into operation.

\subsection{Procedure for Finding an Initial Solution for the Caset Algorithm}

Let $\bar{x}=(\bar{w}, \bar{z}, \bar{y})$ be a solution of the GLCP.

(i) If for each $i=1, \ldots, n$, at least one of the variables $z_{i}$ or $w_{i}$ is nonbasic, then use $\bar{x}$ as the initial solution for the algorithm CASET.

(ii) If there is a pair of complementary variables $\left(\bar{z}_{i}, \bar{w}_{i}\right)$, where both of them are zero and one is superbasic, change the status of this variable with a nonbasic variable $y_{j}$ or a $z_{j}$ (or $w_{j}$ ) variable from a nonbasic complementary pair $\left(\bar{z}_{j}, \bar{w}_{j}\right), j \neq i$. Repeat this procedure until there are no such complementary pairs.

(iii) If there is a zero basic complementary variable (say $z_{i}$ ) whose complementary variable $w_{i}$ is basic or superbasic, perform pivot steps to make $z_{i}$ nonbasic. These pivot steps should maintain as nonbasic all the variables whose complementary variables are basic or superbasic. If such pivot steps are possible, repeat until the situation (i) occurs. Otherwise, the procedure terminates with a failure.

Another difficulty may arise when the solution $\bar{x}=(\bar{w}, \bar{z}, \bar{y})$ found by the algorithm CASET has a complementary pair $\left(\bar{z}_{i}=0, \bar{w}_{i}=0\right)$, such that one of the constraints, say $z_{i} \geq 0$, is considered to be inactive ( $z_{i}$ is basic or superbasic) and the other active ( $w_{i}$ is nonbasic). The following example illustrates this case:

$$
\begin{array}{ll}
\min & f\left(w_{1}, w_{2}, z_{1}, z_{2}, y\right)=w_{2}-y \\
\text { s.t. } & {\left[\begin{array}{l}
w_{1} \\
w_{2}
\end{array}\right]=\left[\begin{array}{cc}
0 & -1 \\
-1 & 0
\end{array}\right]\left[\begin{array}{l}
z_{1} \\
z_{2}
\end{array}\right]+\left[\begin{array}{l}
1 \\
1
\end{array}\right] y+\left[\begin{array}{l}
-1 \\
-1
\end{array}\right]} \\
& z_{i} \geq 0, \quad w_{i} \geq 0, \quad i \in\{1,2\}, \quad 0 \leq y \leq 2, \\
& z_{i} w_{i}=0, \quad i \in\{1,2\} .
\end{array}
$$

If the algorithm CASET is applied to problem (19) using an initial basic solution with basis

$$
\begin{aligned}
& w_{1} \quad y_{1} \\
& B=\left[\begin{array}{ll}
1 & -1 \\
0 & -1
\end{array}\right]
\end{aligned}
$$

associated with the variables $w_{1}$ and $y$, then the following solution is obtained:

$$
w=0, \quad z=0, \quad y=1 .
$$

Furthermore, the corresponding multipliers are

$$
\lambda^{w}=0, \quad \lambda^{z}=\left[\begin{array}{ll}
-1 & 0
\end{array}\right]^{T}, \quad \lambda^{y}=0 .
$$


This shows that there exists a pair of complementary variables $\left(z_{1}, w_{1}\right)$, both zero, with $z_{1}$ being nonbasic and $w_{1}$ basic. Therefore, $w_{1} \geq 0$ is considered as inactive and the CASET algorithm stops without producing a stationary point for MPEC. As in this case, the Lagrange multiplier $\lambda_{1}^{z}$ (reduced gradient) associated with $z_{1}=0$ is negative and $w_{1}=0$, there is an advantage to increase the value of $z_{1}$. To perform this operation, it is necessary to first make nonbasic the variable $w_{1}$. If this operation can be performed, then $z_{1}$ can next be increased from zero without destroying the complementarity of the solution. So the following procedure can be used after the CASET algorithm terminates with a degenerate solution.

\subsection{Improvement Step}

Let $\bar{x}=(\bar{w}, \bar{z}, \bar{y})$ be a degenerate solution found by the algorithm CASET. If there exists a complementary pair with nonbasic $w_{i}=0$ (or $\left.z_{i}=0\right)$ and basic or superbasic variable $z_{i}=0$ (or $w_{i}=0$ ) such that $\lambda_{i}^{w}<0\left(\lambda_{i}^{z}<0\right)$ for $i \in\left(L_{z} \cup L_{w}\right) \backslash\left(L_{z} \cap L_{w}\right)$ make this latter variable nonbasic by pivot steps in such a way that no nonbasic variable, whose complementary variable is either basic or superbasic, is allowed to become basic. Repeat the procedure if necessary.

If we solve problem (19) by the algorithm CASET with this extension, then the solution $w=0, z=\left[\begin{array}{ll}1 & 1\end{array}\right]^{T}$ and $y=2$ is obtained and the corresponding multipliers are given as follows: $\lambda^{w}=\left[\begin{array}{ll}0 & 1\end{array}\right]^{T}, \lambda^{z}=0, \lambda^{y}=1$.

The objective function associated with this solution has a smaller value than the corresponding solution presented in (20). Actually, this solution is a global minimum for MPEC, as the Lagrange multipliers associated with the active constraints are nonnegative.

It is important to point out that this modification might fail when the restricted basis entry rule blocks the process from proceeding.

\section{Computational Experience}

In this section we present some computational experience to exhibit the efficacy of the complementarity active-set algorithm (CASET) described in this paper for obtaining a strongly stationary point for MPEC, as well as to compare this algorithm with some existing methods in the literature. All computations have been performed on a Pentium IV $2.4 \mathrm{GHz}$ machine having $256 \mathrm{MB}$ of RAM.

\subsection{Test Problems}

The test problem generator for linear and quadratic bilevel problems used in our computations was developed by P. Calamai and L. Vicente [11, 17] and allows the generation of problems having known optimal solutions. With this generator [11], it is also possible to perform modifications in the test problems to obtain new instances that have precisely the same characteristics and solutions as those for the initial linear bilevel problems (LBPs), but are less sparse. In this paper, modifications for the three first problems were performed to generate three new LBPs. These are denoted with the same name as the original problem with the addition of the letter "M" (LV1M, 
LV2M, and LV3M, respectively). For creating these modifications, the parameters described in [11] were used.

For additional test problems, we generated also five quadratic bilevel problems QBPs [17] assuming that the feasible region is restricted to the nonnegative orthant, i.e., $x \geq 0$ and $y \geq 0$. This modification does not alter the set of local and global minima for these test problems.

As a second class of test problems, we considered the NP-hard LCPs (linear complementarity problems) that are associated with knapsack problems [12]. The LCPs can be transformed into MPECs using the transformations described in [12].

\subsection{Determination of a Strongly Stationary Point for MPEC}

Since the MPECs associated with the foregoing collection of test problems can be degenerate, it becomes necessary to incorporate the extensions recommended in Sect. 4. In particular, we focus on the improvement step of CASET, which is denoted by CASET-I in Table 1 . In this table, $\mathrm{N}$ represents the dimension of the MPEC, $\mathrm{NC}$ and NI are, respectively, the total number of pairs of complementary variables and iterations performed by CASET, $\mathrm{T}$ is the total CPU time in seconds for solving the MPEC, OBJ. is the value attained for the objective function, and Op.OBJ. is the known optimal value.

Table 1 presents the computational results of Algorithm CASET for solving the MPECs associated with the bilevel problems of Calamai and Vicente $[11,17]$ and the LCPs corresponding to knapsack problems. These results show that, in general, the proposed complementarity active-set algorithm was able to find a strongly stationary point for all problems in a reasonable amount of time and with few iterations. This stationary point is, in some cases, a global minimum for the bilevel problem. For the LCPs associated with knapsack problems, the version CASET-I sometimes achieved better values of the objective function as compared with the original version of CASET with a few more iterations and in the same order of computational effort.

In [7], Leyffer considers a MPEC with optimal solution is $(x, y, w)^{*}=(-1,2,0)$. However, it was shown in [7] that the IPPA [1] converges to a limit point $\left(x^{\infty}, y^{\infty}, w^{\infty}\right)$ with $-.4828 \leq x^{\infty} \leq 0,1 \leq y^{\infty} \leq 1.4828$, and $w^{\infty}=0.0$, which satisfies the nondegeneracy assumption required in [1] for the convergence of the IPPA algorithm, but is not a stationary point of this problem. Analyzing the behavior of the CASET algorithm in solving this problem, we verified that the algorithm indeed achieved a strongly stationary point, which happens to be a global minimum of the problem, in a reduced computational effort.

Fukushima et al. [9] have introduced a sequential quadratic programming algorithm (SQP) for finding a stationary point for an MPEC of the following form:

$$
\begin{array}{ll}
\min & f(x, y), \\
\text { s.t. } & w=q+M x+N y, \quad A y \leq b, \\
& x \geq 0, \quad y \geq 0, \quad w \geq 0, \quad x^{T} w=0,
\end{array}
$$

where $f: \mathbb{R}^{n+m} \rightarrow \mathbb{R}$ is a continuously differentiable function, $N \in \mathbb{R}^{n \times m}, M \in$ $\mathbb{R}^{n \times n}$ is a $P_{0}$ matrix [18], $A \in \mathbb{R}^{p \times m}, q \in \mathbb{R}^{n}, b \in \mathbb{R}^{p}$, and $(x, w, y) \in \mathbb{R}^{2 n+m}$. We 
Table 1 Performance of the CASET algorithm for the LCP and bilevel problems

\begin{tabular}{|c|c|c|c|c|c|c|c|c|c|c|}
\hline & \multirow[t]{2}{*}{ PROB } & \multirow[t]{2}{*}{$\mathrm{NC}$} & \multirow[t]{2}{*}{$\mathrm{N}$} & \multicolumn{3}{|c|}{ CASET } & \multicolumn{3}{|c|}{ CASET-I } & \multirow[t]{2}{*}{ Ор.ОВЈ. } \\
\hline & & & & NI & $\mathrm{T}$ & Овл. & NI & $\mathrm{T}$ & Овј. & \\
\hline \multirow[t]{11}{*}{ LBP } & LV1 & 12 & $12 \times 28$ & 8 & 0.02 & 8.000 & 8 & 0.03 & 8.000 & 6.000 \\
\hline & LV1M & 12 & $12 \times 28$ & 10 & 0.00 & 6.000 & 10 & 0.03 & 6.000 & 6.000 \\
\hline & LV2 & 30 & $30 \times 70$ & 20 & 0.00 & 20.000 & 20 & 0.02 & 20.000 & 16.000 \\
\hline & LV2M & 30 & $30 \times 70$ & 21 & 0.02 & 16.000 & 21 & 0.01 & 16.000 & 16.000 \\
\hline & Lv3 & 55 & $55 \times 125$ & 35 & 0.00 & 40.000 & 35 & 0.03 & 40.000 & 30.200 \\
\hline & LV3M & 55 & $55 \times 125$ & 36 & 0.00 & 33.200 & 36 & 0.03 & 33.200 & 30.200 \\
\hline & Lv4 & 48 & $48 \times 116$ & 29 & 0.00 & 8.000 & 29 & 0.02 & 8.000 & 6.000 \\
\hline & Lv5 & 48 & $48 \times 116$ & 31 & 0.00 & 16.000 & 31 & 0.00 & 16.000 & 14.000 \\
\hline & Lv6 & 110 & $110 \times 250$ & 53 & 0.02 & 12.000 & 53 & 0.02 & 12.000 & 7.500 \\
\hline & LV7 & 110 & $110 \times 250$ & 57 & 0.02 & 28.000 & 57 & 0.05 & 28.000 & 23.500 \\
\hline & Lv8 & 190 & $190 \times 410$ & 94 & 0.03 & 56.000 & 94 & 0.05 & 56.000 & 50.500 \\
\hline \multirow[t]{5}{*}{ QBP } & NLV1 & 8 & $8 \times 20$ & 6 & 0.06 & 0.500 & 6 & 0.00 & 0.500 & 0.313 \\
\hline & NLV2 & 16 & $16 \times 38$ & 10 & 0.03 & 1.000 & 10 & 0.00 & 1.000 & 0.593 \\
\hline & NLV3 & 20 & $20 \times 50$ & 16 & 0.02 & 1.000 & 16 & 0.02 & 1.000 & 0.790 \\
\hline & NLV4 & 60 & $60 \times 145$ & 50 & 0.01 & 1.250 & 50 & 0.00 & 1.250 & 1.040 \\
\hline & NLV5 & 80 & $80 \times 190$ & 57 & 0.03 & 3.250 & 57 & 0.02 & 3.250 & 2.436 \\
\hline LCP - & 1NA1 & 44 & $44 \times 110$ & 40 & 0.02 & 0.499 & 41 & 0.02 & 0.280 & 0.000 \\
\hline \multirow[t]{11}{*}{ KNAPSACK } & 1NA2 & 104 & $104 \times 260$ & 120 & 0.05 & 0.500 & 121 & 0.03 & 0.440 & 0.000 \\
\hline & 1NA3 & 204 & $204 \times 510$ & 203 & 0.05 & 0.346 & 203 & 0.05 & 0.346 & 0.000 \\
\hline & 1NA4 & 304 & $304 \times 760$ & 295 & 0.08 & 0.321 & 295 & 0.09 & 0.321 & 0.000 \\
\hline & 1NB 1 & 44 & $44 \times 110$ & 12 & 0.03 & 0.499 & 13 & 0.02 & 0.310 & 0.000 \\
\hline & $1 \mathrm{NB} 2$ & 104 & $104 \times 260$ & 59 & 0.05 & 0.353 & 59 & 0.02 & 0.353 & 0.000 \\
\hline & 1NB3 & 204 & $204 \times 510$ & 111 & 0.06 & 0.263 & 111 & 0.03 & 0.263 & 0.000 \\
\hline & $1 \mathrm{NB} 4$ & 304 & $304 \times 760$ & 168 & 0.10 & 0.500 & 169 & 0.05 & 0.200 & 0.000 \\
\hline & $1 \mathrm{NC} 1$ & 44 & $44 \times 110$ & 9 & 0.08 & 0.497 & 10 & 0.00 & 0.030 & 0.000 \\
\hline & $1 \mathrm{NC} 2$ & 104 & $104 \times 260$ & 35 & 0.01 & 0.425 & 35 & 0.02 & 0.425 & 0.000 \\
\hline & $1 \mathrm{NC} 3$ & 204 & $204 \times 510$ & 63 & 0.05 & 0.318 & 63 & 0.01 & 0.318 & 0.000 \\
\hline & $1 \mathrm{NC} 4$ & 304 & $304 \times 760$ & 96 & 0.05 & 0.500 & 97 & 0.03 & 0.080 & 0.000 \\
\hline
\end{tabular}

conducted a small computational experiment using some test problems defined in [3] and [9], which have been solved by alternative methods. Table 2 displays the results achieved by using the CASET algorithm, the SQP algorithm described in [9], and the As method (approached stationary point—differentiable method) introduced in [3] for solving these problems. In this table, P.FUK. and P.FAC. represent the particular test problems given in [9] and [3], respectively, while $(m, n, p)$ represents the dimension of the problem. Also, of the two values presented for the number of iterations in the SQP algorithm, the first one is obtained using the same parameter values for the SQP program in the three test problems, while the value in the brackets is achieved with specifically chosen parameter values for each problem. The results 
Table 2 Comparison between the CASET, SQP and AS algorithms

\begin{tabular}{|c|c|c|c|c|c|c|c|c|}
\hline \multirow[t]{2}{*}{ P.FUK. } & \multirow[t]{2}{*}{ P.FAC. } & \multirow[t]{2}{*}{$(m, n, p)$} & \multicolumn{2}{|l|}{ As } & \multicolumn{2}{|l|}{$\underline{\text { SQP }}$} & \multicolumn{2}{|c|}{ CASET } \\
\hline & & & NI & Овл. & NI & Овл. & NI & Овл. \\
\hline 1 & 6 & $(1,1,2)$ & 5 & -3266.67 & $22(6)$ & -3266.67 & 2 & -3266.67 \\
\hline 2 & 9 & $(2,2,4)$ & 7 & $0.36 \mathrm{E}-26$ & $48(6)$ & $0.36 \mathrm{E}-26$ & 4 & $0.25 \mathrm{E}-29$ \\
\hline 3 & 10 & $(4,4,9)$ & 51 & -6600.00 & 31 & -6600.00 & 15 & -6600.00 \\
\hline
\end{tabular}

presented in Table 2 show that, for Problems 1 and 3, all three methods were able to obtain solutions having the same value of the objective function, which is the global minimum of the problem [3]. In Problem 2, CASET has evidently found a strongly stationary point with a better objective value than the one obtained by the other algorithms. Moreover, the CASET algorithm needed only a few of iterations to find these solutions. As the dimension of the problems is small, the computational time is of little significance, and therefore is not presented.

As a main conclusion from this computational experience, we can state that the complementarity active-set algorithm (CASET) is a robust and efficient process to find a strongly stationary point of MPEC. Although it is difficult to establish conclusions regarding comparisons with alternative techniques due to lack of available information, the numerical results generally indicate that the algorithm is competitive with, and sometimes more efficient than, other local methods that have been considered for finding a stationary point for MPEC.

\section{References}

1. Luo, Z., Pang, J., Ralph, D.: Mathematical Programs with Equilibrium Constraints. Cambridge University Press, New York (1997)

2. Outrata, J., Kocvara, M., Zowe, J.: Nonsmooth Approach to Optimization Problems with Equilibrium Constraints: Theory, Applications and Numerical Results. Kluwer Academic, Dordrecht (1998)

3. Facchinei, F., Jiang, H., Qi, L.: A smoothing method for mathematical programs with equilibrium constraints. Math. Program. 85, 107-134 (1999)

4. Fletcher, R., Leyffer, S.: Numerical experience with solving MPECs as NLPs. Technical Report NA/210, Department of Mathematics, University of Dundee (2002)

5. Fukushima, M., Tseng, P.: An implementable active-set algorithm for computing a B-stationary point of a mathematical program with linear complementarity constraints. SIAM J. Optim. 12, 724-739 (2002)

6. Jiang, H., Ralph, D.: Smooth SQP methods for mathematical programs with nonlinear complementarity constraints. SIAM J. Optim. 10, 779-808 (1991)

7. Leyffer, S.: The penalty interior point method fails to converge. Optim. Methods Softw. 20(4-5), 559-568 (2005)

8. Scheel, H., Scholtes, S.: Mathematical programs with complementarity constraints: stationarity, optimality and sensitivity. Math. Oper. Res. 25, 1-22 (2000)

9. Fukushima, M., Luo, Z., Pang, J.: A globally convergent sequential quadratic programming algorithm for mathematical programs with linear complementarity constraints. Comput. Optim. Appl. 10, 5-34 (1998)

10. Scholtes, S.: Active set methods for inverse linear complementarity problems. Judge Institute of Management, Research Paper 28/1999 (1999)

11. Calamai, P., Vicente, L.: Generating linear and linear-quadratic bilevel programming problems. SIAM J. Sci. Comput. 14, 770-782 (1993)

12. Júdice, J., Faustino, A., Ribeiro, I.: On the solution of NP-hard linear complementarity problems. TOP-Sociedad de Estatística e Investigacion Operativa 10, 125-145 (2002) 
13. Bazaraa, M., Sherali, H., Shetty, C.: Nonlinear Programming: Theory and Algorithms, 3rd edn. Wiley, New York (2006)

14. Ribeiro, I.: Global optimization and applications to structural engineering. Ph.D. Thesis, University of Porto, Porto (2005) (in Portuguese)

15. Murtagh, B., Saunders, A.: MINOS 5.0 user's guide. Technical Report SOL 83-20, Department of Operations Research, Stanford University (1983)

16. Gill, P., Murray, W., Saunders, M.: Practical Optimization. Academic, New York (1981)

17. Calamai, P., Vicente, L.: Generating quadratic bilevel programming test problems. ACM Trans. Math. Softw. 20, 103-119 (1994)

18. Cottle, R., Pang, J., Stone, R.: The Linear Complementarity Problem. Academic, New York (1992) 